\title{
Colonic obstruction secondary to incarcerated transiliac bone hernia
}

\author{
Charalampos Seretis ${ }^{1}$, Kalimuthu Marimuthu ${ }^{1}$, and Balasubramaniam Piramanayagam ${ }^{1}$ \\ ${ }^{1}$ George Eliot Hospital NHS Trust
}

October 27, 2020

\begin{abstract}
Herniation of intra-abdominal viscera through pelvic bone defects represents an extremely uncommon complication following orthopaedic interventions in the bony pelvis. We report a rare case of acute colonic obstruction through a defect in the iliac bone, following bone graft harvesting from the iliac crest, aiming to raise clinical awareness.
\end{abstract}

Title: Colonic obstruction secondary to incarcerated transiliac bone hernia

Authors: Charalampos Seretis, Kalimuthu Marimuthu, Balasubramaniam Piramanayagam

Affiliation: Department of General Surgery, George Eliot Hospital NHS Trust. Address: College Street, Nuneaton, Warwickshire, Nuneaton, CV10 7DJ, United Kingdom

Corresponding author: Charalampos Seretis, MSc, DU, Specialty Registrar in General Surgery, George Eliot Hospital NHS Trust. Email: charalampos.seretis@geh.nhs.uk, Tel: 07766478856. Postal address: College Street, Nuneaton, Warwickshire, Nuneaton, CV10 7DJ, United Kingdom

Conflict of interest: The authors declared no potential conflicts of interest with respect to the research, authorship, and/or publication of this article.

Author contributions: CS: contributed to the clinical data collection and prepared the case report; KM and BP: contributed to the design of the case report presentation and performed the final revision of the manuscript.

Keywords: transiliac; hernia; surgery; emergency

Key Clinical Message: Transiliac bone hernias are a rare cause of intestinal obstruction and high clinical suspicion is required for their diagnosis

\begin{abstract}
Herniation of intra-abdominal viscera through pelvic bone defects represents an extremely uncommon complication following orthopaedic interventions in the bony pelvis. We report a rare case of acute colonic obstruction through a defect in the iliac bone, following bone graft harvesting from the iliac crest, aiming to raise clinical awareness.

\section{Case Description}

A 77-year old Caucasian female patient presented to our acute surgical take with features of gastrointestinal obstruction, comprising of abdominal distension, vomiting and sharp pain localised in the left iliac fossa. She had a complex medical history comprising of congestive heart failure, hypertension, diabetes mellitus and previous right knee and hip replacements, along with extraction of left iliac bone graft for spinal surgery. Clinical examination revealed a moderately distended but soft abdomen, with tenderness in deep palpation in the left iliac fossa; no obvious external hernia was identified. Provisional diagnosis was that of intestinal
\end{abstract}


obstruction and hence an urgent abdominopelvic computed tomography (CT) scan with intravenous contrast was performed for further assessment. The performed CT scan revealed the presence of closed loop colonic obstruction between a competent ileoceal valve and the distal descending colon, which had herniated through the left iliac bone, with no obvious evidence of intestinal ischemia or perforation (Figures $1 \& 2$ ). The patient was scheduled for emergency laparotomy and intraoperatively there was no concern regarding the viability of the colon, after the reduction of the herniated segment. The defect was closed with the use of synthetic polypropylene mesh and no colonic resection was eventually required. The patient had an uneventful recovery and was discharged in a stable condition, with no evidence of recurrence at 6 months postoperatively.

Our case highlights the need for high clinical suspicion of a transiliac bone hernia in patients with previous relevant orthopaedic interventions, who present with features of gastrointestinal obstruction, as highlighted by the previous very limited reports in the literature ${ }^{1,2}$. Since clinical examination is usually insufficient to establish the diagnosis, we advocate urgent performance of abdominopelvic computed tomography for diagnostic and pre-operative planning purposes, followed by prompt surgical intervention, with liberal use of prosthetic mesh to reduce the chance of recurrence of the hernia.

Acknowledgments: None to be declared by the authors

\section{References}

Radais F, Facy O, Ortega-Deballon P. Transiliac hernia. Am J Surg. 2011 Jun; 2016: e41-42. doi: 10.1016/j.amjsurg.2010.06.030

Kunin N, Gancel CH, Foret A, Gayet C, Letoquart JP, Daaboul M. Transiliac hernia after bone graft. J Visc Surg. 2013 Dec; 150(6): 419-420. doi: 10.1016/j.jviscsurg.2013.09.007.

\section{Figure Legends}

Figure 1: Axial view of the preoperative CT scan showing the site of the incarcerated transiliac bone hernia, with part of the descending colon protruding through the defect (red arrow). Note the presence of cecal dilatation (yellow arrow), which in absence of small bowel dilatation, was suggestive of closed loop colonic obstruction and mandated urgent laparotomy

Figure 2: Sagittal view of the preoperative CT scan showing the site of the incarcerated transiliac bone hernia (red arrow), with evident proximal colonic obstruction (yellow arrow) 


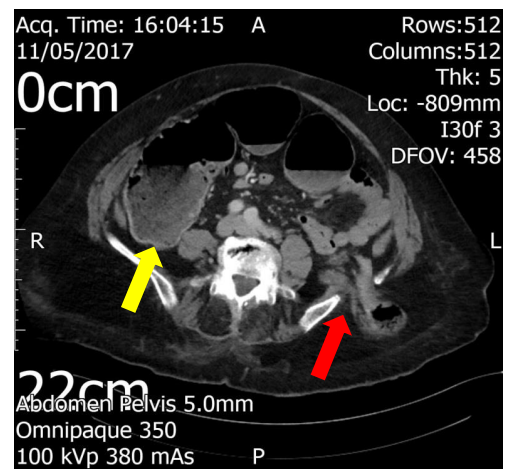

Figure 1: Axial view of the preoperative CT scan showing the site of the incarcerated transiliac bone hernia, with part of the descending colon protruding through the defect (red arrow). Note the presence of cecal dilatation (yellow arrow), which in absence of small bowel dilatation, was suggestive of closed loop colonic obstruction and mandated urgent laparotomy

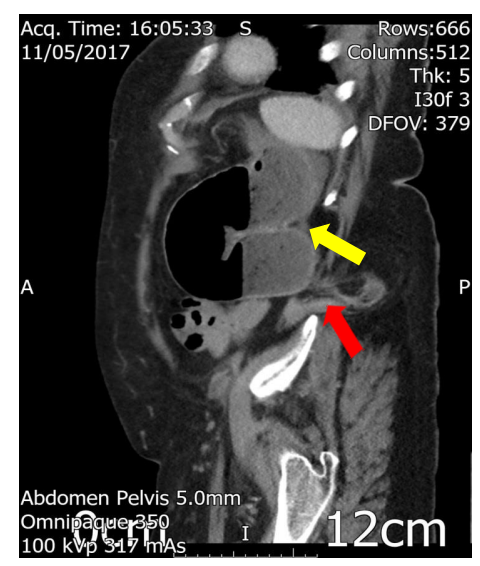

Figure 2: Sagittal view of the preoperative CT scan showing the site of the incarcerated transiliac bone hernia (red arrow), with evident proximal colonic obstruction (yellow arrow) 